\title{
UAV Aided Intelligent Routing for Ad-Hoc Wireless Network in Single-area Theater*
}

\author{
Daniel Lihui Gu, Guangyu Pei, Henry Ly, Mario Gerla, Beichuan Zhang and Xiaoyan Hong \\ Computer Science Department \\ University of California, Los Angeles \\ \{gu,pei,henryly,gerla,bzhang,hxy\}@cs.ucla.edu
}

\begin{abstract}
Large homogeneous ad hoc wireless networks have a problem: the bandwidth available to an mobile user decreases as the number of nodes in the network increases. Using the embedded ad-hoc networking mechanism, nodes are able to transport packets across the network in a multihop fashion. An embedded mobile backbone is dynamically constructed to form 2-level physical heterogeneous multihop wireless network. These backbone nodes provide two critical functions: (1) direct communication between neighboring cluster heads. (2) efficient route discovery in HSR. With the broadcast feature of UAV, Link state can be broadcasted to backbone nodes instead of "flooding" on the level 2. Thus, routing overhead can be tremendously reduced, throughput will be improved. We modified Hierarchical State Routing to have an intelligent selection algorithm to reduce the system latency caused by long propagation delay of UAV channel. The performance of the system is evaluated through simulation experiments.
\end{abstract}

\section{Introduction and Background}

Ad hoc wireless networks are an ideal technology to deploy an instant communication network for civilian and military applications. However, as the size of an ad hoc multi-hop network increases (as in battlefield applications), the available bandwidth to the mobile users decrease. Key causes of such a degradation include the resulting excessive control traffic overhead required to maintain accurate routing tables in the presence of mobility, and the difficulty in guaranteeing any kind of bandwidth on a path with many wireless hops. The latter is of particular concern for the support of real time applications.

There are many existing routing protocols for ad hoc wireless network. They can be classified into four different types: (1) global, precomputed routing. (2) on-demand routing. (3)

\footnotetext{
*This work was supported in part by NSF under contract ANI-9814675, in part by DARPA under contract DAAB07-97-C-D321 and in part by Intel.
}

location based routing. (4) flooding. These approaches all assume that the network is a homogeneous one. All nodes in homogeneous network have the same transmission capabilities and use the same frequency and channel access scheme. Among those four, on demand routing is the most recent one to enter into scalable wireless routing class. By relaxing the requirement of routes maintenance on all nodes, on demand routing avoids excessive routing overhead. In a word, a route to a specific destination is constructed only when needed. However, on demand routing does not scale well to large network when traffic is heavily loaded. As discussed in [2], traffic load will effect routing overhead. In the case of 100 nodes and 40 sources, the results in [2] explain that on demand routing protocols will generate much higher routing overhead than actual throughput capacity. Therefore, the maximum achievable throughput in the simulation scenarios is only 2$3 \%$ of total network capacity [2]. Recent theoretical study in [4] presents the throughput bounds of homogeneous wireless networks. The throughput furnished to each end user eventually reduces to zero as the size of network is progressively increased. The limitation is fundamentally due to the spatial concurrency constraints on nearby nodes sharing the same channel. All these results show us that homogeneous structure can not be scalable to large size ad hoc wireless network. "heterogeneous" hierarchical structure should be the solution.

In this paper we propose a 2-level physical hierarchical structure and address the routing issue in this heterogeneous environment. On the first level, we have regular ground ad hoc wireless network. On top of that, we propose an embedded ad hoc networking mechanism to dynamically construct a point-to-point embedded mobile backbone network as the second level. The mobile backbone network will connect (using separate frequencies from the ground radio network) all backbone nodes using directive antennas to serve a single area (say, a few kilometers in diameter). With these 2-level architecture, backbone nodes are able to transport packets across the regular ground ad hoc wireless network through point-to-point direct wireless links in one hop. We 
implemented a hierarchical routing protocol - HSR [7] to this heterogeneous, hierarchical environment. Equipped with multiple communication interface, backbone nodes establish multi-level communication at different level. Thus, the virtual paths between cluster heads in homogeneous HSR are replaced by wireless direct point-to-point link in heterogeneous HSR. Route discovery is also multi-level. On the level 1 , link state exchanges locally within cluster. On the level 2, link state exchange globally among all cluster heads. Furthermore, backbone nodes are integrated with Unmanned Aerial Vehicle (UAV) through the UAV access net. In each single area, there is a UAV stationed at about $60 \mathrm{kft}$ high as a multifunctional gateway. Since UAV flies at relatively high elevations, thus can be in sight of all the mobile host in the single area to provide communication on-the-move for all fighting units on the ground. This enable two hop transmissions between any pair of ground mobile backbone nodes in the area using UAV as a router in case of wireless direct point-to-point link on the ground got disconnected due to hills or high buildings.

We modify HSR in 2-level heterogeneous environment further to have intelligent capability to use UAV. With the third interface, backbone nodes will be able to send link state information to UAV, then UAV broadcasts to all ground backbone nodes to update routing table simultaneously. The third interface can also be used for data transmition. Intelligent Hierarchical State Routing (IHSR) makes ground backbone nodes to have an option to send packets through UAV access Net if the number of hops on the level 2 is large than a certain number.

The rest of the paper is organized as follows. In section 2, we introduce the infrastructure of 2-level heterogeneous ad hoc wireless network with UAV. Section 3 describes the intelligent hierarchical state routing scheme for heterogeneous environments. Performance evaluation is presented in section 4 and we conclude our paper in section 5 .

\section{2-level Heterogeneous Ad-Hoc Wireless Network with UAV}

\subsection{Architecture of the 2-level Heterogeneous Ad- Hoc Wireless Network}

Figure 1 shows the architecture of a 2-level heterogeneous ad hoc wireless network with UAV. The hierarchical infrastructure consists of the following two hierarchies:

1. level 1: Ground Ad-Hoc Wireless Network: Based on the hop distance of packet transfers, wireless networks

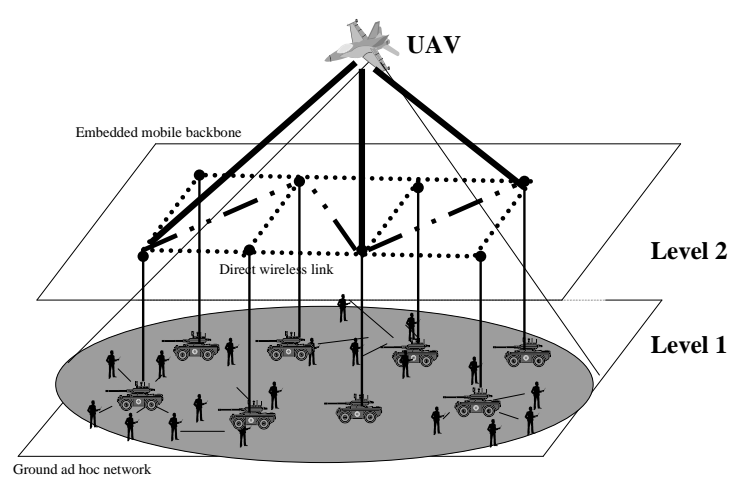

Figure 1: 2-level UAV Heterogeneous Ad-Hoc Wireless Network for Single-area Theater

can be divided into two types: single-hop and multihop. The multi-hop wireless network, also called "ad hoc" wireless network, allows all mobile hosts to move freely without any constraints by fixed communication infrastructure. Due to the ad hoc topology, maintaining efficient routes become very challenging.

At this level, we have both regular ground mobile nodes and backbone nodes. A variety of clustering algorithms have been proposed for the dynamic creation of clusters and the election of cluster heads in ad hoc wireless networks [13]. The only modification needed here is, that backbone nodes will be selected as cluster heads by default. Spread-spectrum radios permit code division multiple access (CDMA) and spatial reuse across clusters. Within a cluster, we use 802.11 as the Medium Access Control (MAC) layer protocol.

2. level 2: Ground Embedded Mobile Backbone network: Due to the poor performance of ad hoc wireless network where many hops are involved, an embedded mobile backbone was introduced. In the tactical environment, special fighting units like trucks, tanks may carry a lot more equipment than individual soldiers. These mobile nodes, with the help of beam-forming antennas, can offer high-speed point-to-point direct wireless links. So if we select those mobile nodes as backbone nodes, we can establish a ground mobile backbone embedded within the ground ad hoc wireless network.

In this level, we only have ground backbone nodes. Direct point-to-point wireless links are used for the communications among the neighboring backbone nodes. 


\subsection{Architecture of UAV Access Net}

In each single-area, there is a UAV maintains a station at an altitude of 50 to 60 thousands feet by flying in a circle with a diameter of around 8 nautical miles. With the help of Phased Array Antennas, it can provide the shared beam to the ground to keep line-of-sight connectivity for one area of operation down below. UAV access net is used for communications from UAV to its ground mobile backbone nodes and from ground mobile backbone nodes to their associated UAV. All the ground backbone nodes in the same area will access UAV using the MAC layer protocol, Centralized Intelligent Channel Assigned Multiple Access (C-ICAMA) [3]. C-ICAMA is the MAC layer protocol developed for ground backbone nodes to access UAV. C-ICAMA has an intelligent scheduling algorithm, which can dynamically allocate bandwidth for up-link and down-link to fit the instantaneous status of asymmetric traffic.

\section{Hierarchical State Routing in the Het- erogeneous Environment}

\subsection{Hierarchical State Routing Protocol}

HSR [7] [9] [5] is a hierarchical link state based routing protocol. It maintains a multi-level hierarchical topology, where the cluster heads at the lower level form the next higher level and become the members of that level. These new members will organize themselves in clusters on the new level and so on recursively. The purpose of clustering is to reduce the routing overhead and to efficiently use the radio channel resources. HSR provides the multilevel clustering as well as multilevel logical partitioning. Clustering is based on geographical (physical) relationship among nodes, ( so, it is also called physical clustering). Logical partitioning, on the other hand, is based on logical relationship among nodes (e.g. soldiers in the same company). Logical partitions play a major role in mobility management.

\subsection{Intelligent Hierarchical State Routing Protocol}

In this paper, we have developed Intelligent Hierarchical State Routing (IHSR) in a single-area theater to fit tactical requirement. Intelligent HSR (IHSR) would establish multilevel communications with multiple interfaces at different level.

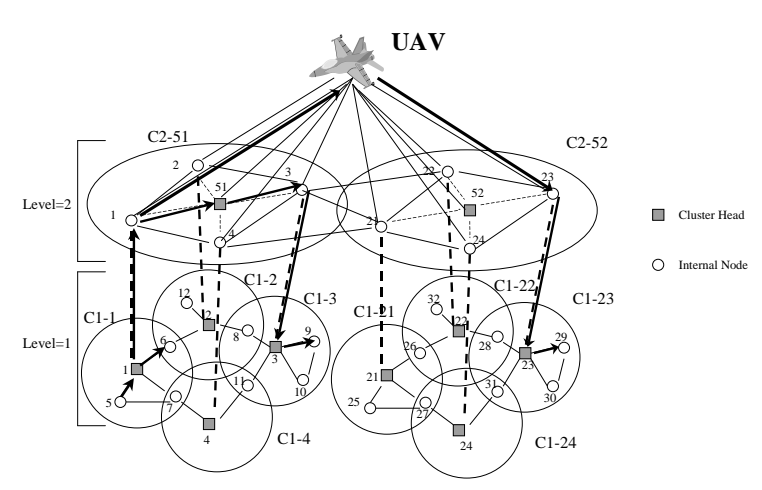

Figure 2: 2-level Physical Hierarchical Clustering in Singlearea Theater

\subsubsection{Physical 2-level Partition}

The key of IHSR routing is to segment network nodes into multi-level clusters. Each cluster contains cluster head and cluster internal nodes. A group of cluster heads form clusters again on the next hierarchical level. The physical 2-level clustering hierarchy used in IHSR is illustrated in Fig. 2. In the level 1 , we use clustering algorithm to dynamically create clusters and elect cluster heads. In the IHSR clustering algorithm, ground backbone nodes will be selected as cluster heads by default. We have 8 physical clusters in each area at this level. Generally, there are two kinds of nodes in a cluster at any level: cluster-head node (e.g., Node 1, 2, 3, 4, 21, 22, 23 and 24), and internal node (e.g., 5, 6, 7, 8, 9, 10, 11, 12, 25, 26, 27, 28, 29 and 30). The cluster-head node acts as a local coordinator of transmissions within the cluster and responsible for keeping and updating routing information beyond the cluster. Thus, internal node only needs to know the local information within cluster. Level 2 consists of all ground backbone nodes which were selected as cluster heads in level 1 . In level 2, one area will have two clusters, Home Agent will be the cluster head. There are two kinds of nodes in a cluster at level 2 : cluster-head node (HA), and internal node (ground backbone nodes). All nodes will communicate to each other via direct point-to-point wireless links. When they need to use UAV, they will communicate with UAV through shared beam.

As part of the physical 2-level partition, the concept of HID (Hierarchical ID) is introduced in IHSR as the same in HSR [5]. Each node has a unique identifier NodeID. NodeIDs are the physical hardwired addresses (i.e., MAC ad- 
dresses). The NodeIDs shown in Fig. 2 are MAC addresses. In IHSR, we define the HID (Hierarchical ID) of a node as the sequence of the MAC addresses of the nodes on the path from the top hierarchy to the node itself.

\subsubsection{Logical Partition}

In order to have better mobility management, the concept of logical addresses is introduced in IHSR in addition to MAC addresses. Logical addresses has two fields (subnetID, hostID). These addresses have a pattern similar to IP, and can be viewed as private IP addresses for the entire area. Each IP subnet defines a particular user group with similar features (e.g., tank battalion in the battlefield, soldiers in the same company). The transport layer delivers to the network a packet with the tactical private IP address. The network will resolve the IP address into a HID which is based on MAC addresses. The notion of subnet is important because each subnet is associated with a home agent. All home agent will advertise their HIDs to the top hierarchy (UAV). Thus, the home agent HIDs are appended to the top level routing tables on UAVs. When a source node wants to deliver a packet to a destination node of which it knows the IP address, it first take the subnet address field from the IP. From its internal list (or top hierarchy) it gets the hierarchical address of the corresponding home agent. It then sends the packet to the home agent with this HID. The home agent will find the registered address from the host ID of the IP address and sends the packet to the destination. Once source node and destination node have discovered each other's HID, packets can be sent directly without involving the home agent.

\subsubsection{Route Discovery}

1. Routing Fields: In IHSR, route discovery is conducted on different hierarchical level. Link state messages are exchanged locally among internal nodes within each cluster and globally among cluster heads through neighboring cluster heads exchange and UAV broadcasts. Fig 3 . shows the data structures of IHSR routing fields on different nodes.

The logical address is the network address that is assigned to this node, which contains the subnet ID and host ID. The HID field is a tuple of three node IDs, which contains the ID of current cluster head this node associated with. Home Agent address is the logical address of the Home Agent that node belongs to. The level field stores the hierarchical level information of that node. All these information will be recorded on

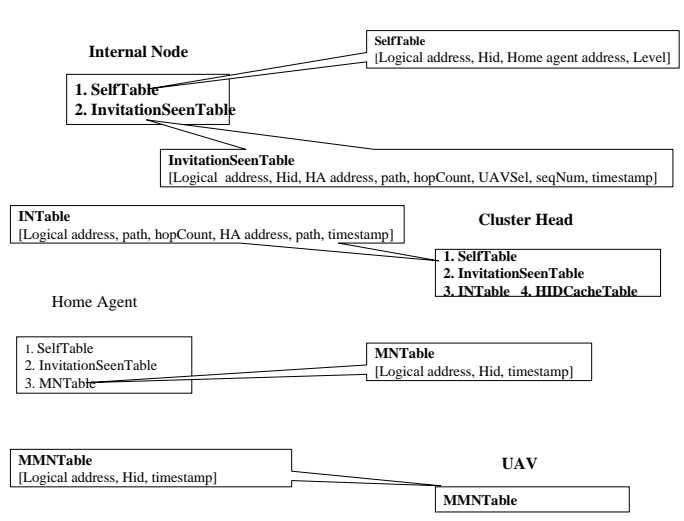

Figure 3: IHSR Routing Field

SelfTable for all ground nodes.

Cluster heads periodically broadcast out invitation messages to the network. The InvitationSeenTable records the invitation message that the node has seen so far. Each InvitationSeenTable entry keeps sufficient information to route packets to the destination cluster head. Since there is a timestamp value in each entry, the entry will be flushed if there is no invitation message received for a certain amount of time.

All internal nodes have both SelfTable and InvitationSeenTable as their routing table. If a node become cluster head, it will have two tables on it's routing table: INTable and HIDCacheTable. INTable, stands for internal node table, is used to record the information of internal nodes within this cluster. This is how the cluster head know the current internal nodes inside the cluster. When cluster head receive registration message from it's internal nodes, it will add one entry on this table. If no registration received for a period of time, the existing entry on the table will be removed. HIDCacheTable is used for routing caching only. Each cluster head keeps a cache of HID it seen most recently and selection of using UAV or not. If there is a cache hit, packets will be routed directly to the destination without referencing Home Agent (if destination is an internal node of other cluster). Home Agent will have MNTable, which is responsible for name resolving between logical address and hierarchical address for all members inside the subnet. UAV, with MMNTable, is responsible for name resolving for all members of multiple subnet in the single-area theater. 
2. Invitation Message: In IHSR, the route discovery is based on link state exchange of invitation message. However, the link state exchange in multi-level heterogeneous environment is totally different from homogeneous environment. Link state exchange here is conducted in 3 different ways. (1) Link state exchange is localized within the cluster, since only cluster heads send out Invitation Messages. (2) Link state "flooding" on level 2 is limited on certain number of hops. (3) For any long hops link state exchange, UAV will broadcast it to the entire single-area.

3. Registration Message: In respond to Invitation Message, registration message is replied directly to the senders. Lower level nodes send registration message in respond to invitation message from the upper level. Upon receiving registration message, cluster head record the node as it's internal node by storing the information in INTable.

4. Routing: HID is used to route data packets in IHSR if UAV is not selected. From invitation registration message exchange, cluster head has the knowledge of both local information (within cluster) and global information (within single-area). Locally, it knows it's internal nodes from INTable lookup. Globally, it knows all the other cluster head from InvitationSeenTable lookup. The following three examples show how packets are routed in IHSR for three different cases. ( see fig 2)

- Example 1: Source N5 to destination N6

(a) N5 sends packet to N1

(b) N1 searches INTable, N6 is it's internal node.

(c) N1 forwards packet to N6

- Example 2: Source N5 to destination N9

(a) N5 sends packet to $\mathrm{N} 1$

(b) N1 searches INTable, N9 is not an internal node

(c) N1 searches InvitationSeenTable and finds Home Agent N51 and sends to N51

(d) N51 searches MNTable and finds N9 is located at cluster of $\mathrm{N} 3$ and sends $\mathrm{N} 3$

(e) N3 forwards to N9

(f) N3 responds to N1 and N1 caches Hid of N9 for later packets

- Example 3: Source N5 to destination N29

(a) N5 sends packet to N1 (b) N1 searches INTable, N29 is not an internal node

(c) N1 searches InvitationSeenTable and finds Home Agent N51 and sends to N51

(d) N51 searches MNTable and finds N29 is not the member of this subnet

(e) N51 forwards to UAV and responds to N1 and N1 cache UAVSel of N29 in it's HIDCacheTable for later packets

(f) UAV searches MMNTable and finds N29 is located at subnet of N52 and sends to N52

(g) N52 searches MNTable and finds N29 is located at cluster of N23 and sends to N23

(h) N52 responds to UAV and UAV caches Hid of $\mathrm{N} 29$ for later packets

(i) N23 searches INTable, N29 is it's internal node

(j) N23 forwards packet to N29

\section{Performance Evaluation}

Our simulation environment is the GlomoSim library 1.2.3 [10] written in the parallel, discrete-event simulation language PARSEC [1]. The ground radios model reflects commercial radios, such as Lucent's WaveLAN. The data rate is 2 Mbps. The transmission range is 150 meters. The MAC layer protocol used among ground radios is IEEE802.11. Each ground backbone node has three different physical interfaces: (1) ground radio interface, which is used for communications among regular ground nodes and from regular ground nodes to backbone nodes; (2) directional point-to-point wireless links among backbone nodes and (3) radio interface for accessing UAV aerial backbone nodes. The MAC protocol for backbone node to access UAV is Centralized Intelligent Channel Assigned Multiple Access (C-ICAMA) [3].

We have compared HSR in heterogeneous environment with an ideal routing protocol in which routes are calculated based on the accurate topology provided by the simulator. Ideal routing has no-overhead on routing message exchange, and always has the most accurate knowledge of the entire network topology. This hypothetical protocol represents the performance upper bound for all possible routing protocols. The simulation results show that HSR in a heterogeneous environment can outperform ideal routing protocol in a homogeneous environment. Due to the long propagation delay of accessing UAV, the averay system latency will be increased in this environment. However, with the intelligent selection, 
route can select either UAV channel or direct point-to-point wireless link to retrieve higher throughput as well as lower system delay. Due to the page limitation here, the detail simulation results will be available in conference presentation slides.

\section{Conclusion}

We have introduced the Intelligent Hierarchical State Routing (IHSR) in hierarchical, heterogeneous 2-layer ad hoc wireless networks. The IHSR is the implementation of the previously proposed HSR in 2-layer heterogeneous environment with the intelligent selection of using UAV for single-area theater. It improves scalability by reducing the number of transmissions with the help of hierarchical 2-layer infrastructure. It improves throughput for link state routing by using UAV broadcasts link state instead of "flooding".

\section{References}

[1] R. Bagrodia, R. Meyer, M. Takai, Y. Chen, X. Zeng, J. Martin, and H.Y. Song, "PARSEC: A Parallel Simulation Environment for Complex Systems," IEEE Computer, vol. 31, no. 10, Oct. 1998, pp.77-85.

[2] S.R. Das, C.E. Perkins and E. M. Royer, "Performance Comparison of Two On-demand Routing Protocols for Ad Hoc Networks", In Proceedings of IEEE INFOCOM 2000, Tel Aviv, Israel, Mar. 2000, pp. 3-12

[3] Daniel Lihui Gu, Henry Ly, Xiaoyan Hong, Mario Gerla, Guangyu Pei and Yeng-Zhong Lee "C-ICAMA, A Centralized Intelligent Channel Assigned Multiple Access for Multi-Level Ad-Hoc Wireless Networks with UAVs", To appear in Proceedings of IEEE WCNC 2000, Chicago, IL, Sep. 2000.

[4] P. Gupta and P. R. Kumar, "The Capacity of Wireless Networks", To appear in IEEE Transactions on Information Theory.

[5] A. Iwata, C.-C. Chiang, G.Pei, M. Gerla, and T.-W. Chen, "Scalable Routing Strategies for Ad-hoc Wireless Networks", IEEE Journal on Selected Areas in Communications, Special Issue on Ad-Hoc Networks, Aug. 1999, pp. 1369-1379

[6] C.-C. Chiang, M. Gerla, and L. Zhang, “ Adaptive Shar ed Tree Multicast in Mobile Wireless Networks," In Proceedings of IEEE GLOBECOM'98,
[7] G. Pei, M. Gerla, X. Hong, and C.-C. Chiang, "A Wireless Hierarchical Routing Protocol with Group Mobility," In IEEE WCNC'99,New Orleans, LA, Sep. 1999, pp. $1536-1540$

[8] D.B. Johnson and D.A. Maltz, "Dynamic Source Routing in Ad Hoc Wi reless Networks," In Mobile Computing, edited by T. Imielinski and H. K orth, Chapter 5, Kluwer Publishing Company, 1996, pp. 153-181.

[9] G. Pei and M. Gerla, "Mobility Management in Hierarchical Multi-hop Mobile Wireless Networks," In Proceedings of IEEE ICCCN'99, Boston, MA, Oct. 1999, pp. 324-329

[10] M. Takai, L. Bajaj, R, Ahuja, R. Bagrodia and M. Gerla, "GloMoSim: A Scalable Network Simulation Environment," Technical report 990027, UCLA, Computer Science Department, 1999.

[11] H.H. (Sam) Khatib. "Theater Wideband Communications.” IEEE MILCOM, page 378, November 1997.

[12] Ram Voruganti and Allen Levesque. " $C^{4}$ I Mobility Architectures for $21^{\text {st }}$ Century Warfighters." IEEE MILCOM, page 665, November 1997

[13] C.-C. Chiang, H-K Wu, Winston Liu, and Mario Gerla. "Routing in Clustered Multi-hop, Mobile Wireless Networks", The IEEE Singapore International Conference on Networks, pp 197-211, 1997 